\title{
Emotional intelligence and its impacts on the clinical performance of nurses in general public hospitals
}

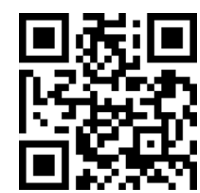

Original article

Deldar Morad Abdulahª̈, Rasoul Sabri Piro ${ }^{b}$, Yousif Ali Yaseenc

${ }^{a}$ Community Health Unit, College of Nursing, University of Duhok, Duhok, Duhok 42001, Iraq

${ }^{b}$ Psychiatric Nursing Unit, College of Nursing, University of Duhok, Duhok, Duhok 42001, Iraq

IInternal Medicine Department, College of Medicine, University of Duhok, Duhok, Duhok 42001, Iraq

Received: 15 April 2021; Accepted: 28 May 2021; Published: 20 December 2021

Abstract: Objectives: The nursing profession is considered to be at a high risk of workplace stress, workload, and burnout due to the high job demands. The nurses require the appropriate and prompt coping mechanisms to relieve the effects of stress. The nurses have to be emotionally intelligent to overcome the work-related stress to meet the clinical-based duties. But there is a paucity of the effect of emotional intelligence (EI) on the clinical performance of nurses in the literature. Therefore, the role of dimensions of El in the improvement of clinical performance of nurses was explored in this study.

Methods: A total of 194 nurses with different education levels and experiences were included from various clinical departments and shifts of public hospitals in Iraqi Kurdistan.

Results: The mean age of the nurses was 29.44 years, with ages ranging from 23 years to 36 years. The nurses were men $(56.70 \%)$, married $(53.10 \%)$, non-smokers $(86.10 \%)$, physically active $(55.70 \%)$, and irregular physically active $(63.90 \%)$. Nurses were able to appraise their own emotions but not the emotions of other nurses. They regulated their own emotions but were weak in regulating the emotions of other nurses. They did not utilize emotions in coming up with new ideas and facing obstacles in clinical settings. Generally, the nurses reported that a little clinical performance is required in medical settings. The levels of interpersonal communication and problem solving were improved with an increasing appraisal and regulating own emotions. The level of problem solving was improved with appraising own emotions and utilizing emotions in facing obstacles.

Conclusions: Nurses need to know how to regulate others' emotions and utilize these emotions in coming up with new ideas and facing the obstacles positively in medical settings.

Keywords: emotional intelligence $\bullet$ clinical performance $\bullet$ communication

(c) Shanxi Medical Periodical Press.

\section{Introduction}

The function of nurses is considered to be a crucial part of health care teams. The reasons for this importance can be attributed to the role of nurses in establishing coordination for health care services and facilitating effective communication between staff, patients, and families. The nursing profession is considered to be at a high risk of workplace stress, workload, and burnout due to the high job demands. The job demands are

How to cite this article: Abdulah DM, Piro RS, Yaseen YA. Emotional intelligence and its impacts on the clinical performance of nurses in general public hospitals. Front Nurs. 2021;4:381-388. 
workloads and health risks linked to direct contact with patients along with a bulk of administrative duties. ${ }^{1}$ In this regard, nurses need to cope with high job-associated stress along with a high clinical burden. These stresses may cause harm and impact nurses' well-being and their abilities to cope with job assignments. More than $40 \%$ of the nurses have moderate to severe levels of workplace stress. ${ }^{2} \mathrm{~A}$ study conducted in this region reported that $83.60 \%$ and $4.50 \%$ of nurses have moderate and high stress levels, respectively. ${ }^{3}$

Given to inherence of the nursing profession, the nurses require the appropriate and prompt coping mechanisms to relieve the effects of stress. The nurses' responses to stressful events in terms of coping mechanisms are different based on individual varieties and organizational factors. Therefore, nurses have to be emotionally intelligent to overcome the work-related stress in order to accomplish the clinical-based duties. ${ }^{4}$

According to the APA Dictionary of Psychology, emotional intelligence $(\mathrm{EI})$ assists a person in processing and using emotional information in reasoning and other cognitive actions. Based on Mayer and Salovey's 1997 model, El includes 4 abilities: identifying and evaluating emotions accurately; accessing and arousing emotions in facilitating cognition; understanding and using emotional language and information; and regulating one's own and others' emotions to promote growth and well-being. ${ }^{5}$

El has received special attention in the nursing profession because it helps nurses to improve their well-being and impacts patients and families. ${ }^{6}$ El skills assist nurses in coping with the emotional demands of the health care environment. Emotional labor is widely accepted as a part of nursing work because nurses empathize highest quality of care for patients and show their humanity. Nurses need to manage their emotions to offer appropriate help and support. Emotions are important in creating and maintaining an appropriate environment to care for patients. ${ }^{7}$ The ability to create a rapport with patients, manage their emotions, and better focus on the patients' needs is essential to provide quality care ${ }^{8}$ Interestingly, a substantial relationship between social skills and clinical competence has been found among nurses. ${ }^{9}$

The existing literature has focused considerably on the relationship between $\mathrm{El}$ and academic performance among nursing students. Academic performance, however, is quite different from job performance. ${ }^{10-12}$ There is a paucity in the literature on the effect of $\mathrm{EI}$ on the clinical performance of nurses. A recent study conducted in this region has reported that nurses have a low level of El. ${ }^{3}$ In this regard, we aimed to examine the relationship between 5 dimensions of El and clinical performance of nurses in this study.
We hypothesized that the levels of problem solving and interpersonal communication are regulated by appraisal of other nurses' emotions and utilization of emotions in facing obstacles in clinical settings.

\section{Methods}

\subsection{Study design and sampling}

Nurses who were working in different hospitals across Duhok city were invited to participate in this crosssectional study. The nurses were invited from different hospital types, clinical departments, and working shifts. The sites were multi-specialty and comprehensive, and they included 1 general, 1 pediatric, 1 maternity, 1 emergency public hospital, and 1 cardiac center. The clinical settings were medical, surgical wards, and out-patient clinics; intensive care units (ICUs); cardiac care units (CCUs); operation rooms; and anesthesia settings of hospitals. The data collection was performed between November 2017 and January 2018.

The purposes of the study were explained to the subjects before filling out the questionnaire, personally or in small groups that included 2 or 3 persons. The data collection was performed in a calm and appropriate environment inside the hospital to avoid staff interruption. The total time required to fill out the questionnaire was only $15 \mathrm{~min}$.

\subsection{Eligibility criteria}

The subjects met the eligibility criteria for the present investigation if they were men or women and if they were aged 18 years and older, regardless of sociodemographic aspects, education levels, and job rank.

The nurses who had an experience of $<6$ months were not included in the study because nurses have a rotating working schedule among different hospitals and clinical departments for the first 6 months of employment in this region. We excluded these nurses from the study due to the lack of stability during this time. Nurses with chronic medical conditions or any mental disorder were also excluded from the study. Therefore, of the 221 subjects invited, 19 nurses did not agree to participate and 8 nurses had $>20 \%$ missing information. Finally, 194 nurses were included in this study (response rate: $87.78 \%)$.

\subsection{Measurement criteria}

The Brief Emotional Intelligence Scale (BEIS-10) was used to measure El. It has been created based on the longer 33-item EI Scale (EIS). The BEIS-10 has 10 items that assess 5 distinct categories of El. The categories 
include the appraisal of their own emotions, appraisal of others' emotions, regulation of their own emotions, regulation of others' emotions, and utilization of emotions. The items are rated on a 5-point Likert scale anchored based on the following scores: 1 = "strongly agree," 2 = "agree," 3 = "neutral," 4 = "disagree," and $5=$ "strongly disagree." The agreement score proportion for the items was from $89.20 \%$ to $96.40 \%$ within a \pm 1 range. 13 The Cronbach's alpha value of the 10 items for internal consistency was 0.753 in this study.

The clinical performance of the nurses was measured by an objective structured clinical examination (OSCE) scale. ${ }^{14}$ It assesses clinical competence in a planned or structured way. This scale was used previously by Ross et al. ${ }^{15}$ to measure clinical skill performance among nurses. Various components are measured by the OSCE, including obtaining clinical data (history taking and physical assessment), analyzing clinical data (problem identification and nursing diagnoses), and using clinical data (decision making and supportive, educative, or therapeutic nursing interventions). Each component is an objective of one of the OSCE stations. In this study, the nurses were asked to rate each item in terms of the extent to which it was important in their particular work environment on a 7-point scale: from 1 = "never required" to 7 = "critical." The Cronbach's alpha value of the 38 items for internal consistency was 0.945 .

The Perceived Stress Scale (PSS) measured the perceived degree of stress experienced by a nurse. The tool has 10 items to determine how unpredictable, uncontrollable, and overloaded responders find their lives. The items ask the nurses about their feelings and thoughts over the last month. The PSS measures a special way that a person feels. Items are rated on a 5-point Likert scale anchored based on the following scores: 0 = "never," 1 = "almost never," 2 = "sometimes," 3 = "fairly often," and 4 = "very often." The scores for questions 4,5 , 7 , and 8 must be reversed as $0=4,1=3,2=2,3=1$, and $4=0$, respectively. Subsequently, the scores of the items are summed to obtain a total score between 0 and 40 . Higher scores indicate higher perceived stress. Scores ranging from $0-13,14-26$, and $27-40$ are perceived as low stress, moderate stress, and high stress, respectively ${ }^{16}$. The Cronbach's alpha value of the 10 items for internal consistency was 0.628 in this study.

The sociodemographic aspects of the nurses, including age, gender, residency, education level, and lifestyle, were collected through the self-report method.

\subsection{Statistical analysis}

The general characteristics of the nurses were presented in terms of means (standard deviation) or numbers (percentages). The levels of El and dimensions of clinical performance were determined by using means (standard deviation). The prevalence of stress was determined by numbers (percentages). The role of the El dimensions in regulating the clinical performance of the nurses was examined in a multivariate analysis model. In the multivariate analysis model, the dimensions of clinical performance were considered as dependent variables, and the dimensions of $\mathrm{El}$ as independent variables. In the multivariate analysis model, the adjustment was made for age, gender, marital status, residency, smoking, physical activity, sleeping hours, family size, and stress score.

The comparison of teamwork scores between nurses with different educational levels and smoking status was examined with an independent $t$-test. In this study, the institute and bachelor certificates meant completing 2 years and 4 years at university, respectively. The correlation of the dimensions of El with clinical performance and stress was examined in a bivariate regression analysis. The analyses in the regression were adjusted for age, gender, marital status, residency, smoking, physical activity, sleeping hours, family size, and stress score. A level of $<0.05$ was considered a statistically significant difference. The Statistical Package for the Social Sciences (SPSS), version 25.00, (IBM SPSS Statistics for Windows, version 25.0; IBM Corp., Armonk, NY, USA) was used for the data analysis.

\section{Results}

The mean age of the nurses was 29.44 years ( $S D=4.01$ years), with ages ranging between 23 years and 36 years. The mean family size was $6.81(S D=3.08)$. More than half of the nurses were men $(56.70 \%)$, married $(53.10 \%)$, living in urban areas $(77.30 \%)$, nonsmokers $(86.10 \%)$, physically active $(55.70 \%)$, and irregular physically active $(63.90 \%)$. The study revealed that $52.10 \%$ of them were normal sleepers (defined as $6-8 \mathrm{~h}$ of sleeping/24 h), followed by $31.40 \%$ of short sleepers (defined as $<6$ h of sleep) and $16.50 \%$ of long sleepers ( $>8 \mathrm{~h}$ of sleep), see Table 1 . The nurses had no chronic diseases or psychiatric disorders, as indicated through a self-report technique.

The mean score of $\mathrm{El}$ in the nurses was 2.56 of 5.0. The nurses appraised their own emotions (mean $[M]=1.95)$, but they were unable to appraise the emotions of other nurses $(M=3.20)$. They enabled to regulate their emotions $(M=2.33)$, but they were unable to regulate the emotions of other nurses $(M=2.59)$. Further, the nurses did not think that they could utilize their emotions in clinical settings $(M=2.72)$. Most of the nurses had a moderate level of stress $(77.80 \%)$, 


\begin{tabular}{|c|c|c|c|c|}
\hline \multirow{2}{*}{ Characteristics } & \multicolumn{4}{|c|}{ Statistics } \\
\hline & M & SD & $n$ & $\%$ \\
\hline Age (23-36 years) & 29.44 & 4.01 & & \\
\hline Family size (2-12 no.) & 6.81 & 3.08 & & \\
\hline \multicolumn{5}{|l|}{ Gender } \\
\hline Male & & & 110 & 56.70 \\
\hline Female & & & 84 & 43.30 \\
\hline \multicolumn{5}{|l|}{ Marital status } \\
\hline Single & & & 91 & 46.90 \\
\hline Married & & & 103 & 53.10 \\
\hline \multicolumn{5}{|l|}{ Residency } \\
\hline Urban & & & 150 & 77.30 \\
\hline Rural & & & 44 & 22.70 \\
\hline \multicolumn{5}{|l|}{ Smoking } \\
\hline Yes & & & 27 & 13.90 \\
\hline No & & & 167 & 86.10 \\
\hline Physically active & & & 108 & 55.70 \\
\hline Regular & & & 39 & 36.10 \\
\hline Irregular & & & 69 & 63.90 \\
\hline \multicolumn{5}{|l|}{ Sleeping hours } \\
\hline$<6 \mathrm{~h}$ & & & 61 & 31.40 \\
\hline $6-8 \mathrm{~h}$ & & & 101 & 52.10 \\
\hline$>8 \mathrm{~h}$ & & & 32 & 16.50 \\
\hline \multicolumn{5}{|l|}{ Education } \\
\hline Institute & & & 121 & 62.40 \\
\hline Bachelor & & & 73 & 37.60 \\
\hline
\end{tabular}

Table 1. General characteristics of nurses $(n=194)$.

followed by those with a low level $(14.90 \%)$ and a high level (7.20\%; see Table 2 ) of stress.

Generally, the nurses reported that a little clinical performance is required $(M=3.42)$. They neither agreed nor disagreed with the contextual behaviors $(M=3.71)$. The nurses reported that professional and clinical skills were rarely required $(M=1.57$ and 1.98 , respectively). The following categories required a little clinical performance, including interpersonal communication $(M=3.65)$, problem solving $(M=4.06)$, professional ethics $(M=4.10)$, teamwork $(M=3.91)$, and leadership $(M=3.76)$ in clinical settings (Table 2$)$.

In the multivariate analysis model, the dimensions of clinical performance were considered as dependent variables, and the dimensions of $\mathrm{El}$ as independent variables. The study showed that teamwork was affected by education level $(P=0.006)$. In other words, the nurses with a bachelor's level of education had a significantly greater mean score for teamwork compared to the nurses with an institute level (4.43 vs. $3.61 ; P<0.001)$.

\begin{tabular}{lcc}
\hline \multirow{2}{*}{ Emotional intelligence and perceived stress } & \multicolumn{2}{c}{ Statistics } \\
\cline { 2 - 3 } & $\mathrm{M}$ & $\mathrm{SD}$ \\
\hline \hline Emotional intelligence, mean (SD) & 2.56 & 0.39 \\
Appraisal of own emotions & 1.95 & 0.71 \\
Appraisal of others' emotions & 3.20 & 0.74 \\
Regulation of own emotions & 2.33 & 0.75 \\
Regulation of others' emotions & 2.59 & 0.82 \\
Utilization of emotions & 2.72 & 0.94 \\
Perceived stress no (\%) & & \\
Low stress & 29 & 14.9 \\
Moderate stress & 151 & 77.8 \\
High stress & 14 & 7.2 \\
Clinical nurse performance, Mean (SD) & 3.42 & 0.42 \\
Contextual & 3.71 & 0.54 \\
Professional skill & 1.57 & 0.23 \\
Clinical skill & 3.91 & 1.30 \\
Interpersonal communication & 3.76 & 0.82 \\
Problem solving & & \\
Professional ethics & 4.06 & 1.11 \\
Teamwork & 4.10 & 0.84 \\
Leadership & & 1.28 \\
\hline
\end{tabular}

Table 2. Emotional intelligence and perceived stress in nurses $(n=194)$.

A commitment to professional ethics was associated with smoking status $(P=0.016)$. In other words, nonsmoking nurses had a significantly higher mean score for professional ethics compared to smokers (4.16 vs. 3.73; $P=0.013$ ) (Tables 3 and 4).

Interpersonal communication and problem solving were correlated with an appraisal of one's own emotions. Moreover, teamwork was associated with the regulation of others' emotions, and problem solving was associated with utilization and stress scores (Table 3). The levels of interpersonal communication and problem solving were significantly improved with an increasing level of appraisal of one's own emotions $(r=-0.263$, $P<0.001$ and $r=-0.241, P=0.001$, respectively). The mean scores of interpersonal communication and teamwork were improved with increasing levels of regulation of others' emotions $(r=-0.591, P<0.001$ and $r=-0.527, P<0.001$, respectively). Moreover, the level of problem solving improved with greater utilization of emotion in clinical settings $(r=-0.358, P<0.001)$. The stress level of the nurses increased with a decreasing level of utilization of emotions $(r=0.368, P<0.001$; Table 5). 


\begin{tabular}{|c|c|c|c|c|c|}
\hline \multirow{2}{*}{ Factors } & \multicolumn{5}{|c|}{ Dependent variables: Dimensions of clinical performance } \\
\hline & Dependent variable & Mean square & F score & $P$-value & Partial eta squared \\
\hline Education & Teamwork & 9.367 & 7.739 & 0.006 & 0.042 \\
\hline Smoking & Professional ethics & 4.254 & 5.872 & 0.016 & 0.032 \\
\hline \multicolumn{6}{|l|}{ Appraisal of own emotions } \\
\hline & Interpersonal communication & 6.162 & 5.787 & 0.017 & 0.032 \\
\hline & Problem solving & 3.522 & 7.642 & 0.006 & 0.041 \\
\hline Appraisal of others' emotions & No factor & & & & \\
\hline Regulation of own emotions & No factor & & & & \\
\hline \multicolumn{6}{|l|}{ Regulation of others' emotions } \\
\hline & Interpersonal communication & 50.812 & 47.725 & 0.000 & 0.212 \\
\hline & Teamwork & 60.925 & 50.336 & 0.000 & 0.221 \\
\hline Utilization of emotions & Problem solving & 4.569 & 9.914 & 0.002 & 0.053 \\
\hline Stress score & Problem solving & 9.868 & 21.413 & 0.000 & 0.108 \\
\hline
\end{tabular}

Note: The multivariate analysis model was performed for statistical analysis.

Table 3. Role of emotional intelligence dimensions on the clinical performance of nurses $(n=194)$.

\begin{tabular}{lrlcl}
\hline Clinical performance & $n$ & Mean & Standard deviation & $P$-value \\
\hline \hline Teamwork score & & & & \\
Education & & & & $<0.001$ \\
$\quad$ Institute & 121 & 3.61 & 1.25 & \\
$\quad$ Bachelor & 73 & 4.43 & 1.23 & \\
Professional ethics & & & & \\
Smoking & & & & \\
$\quad$ Yes & 27 & 3.73 & 0.013 & \\
No & 167 & 4.16 & 0.83 & \\
\hline
\end{tabular}

Note: An independent $t$-test was performed for statistical analyses.

Table 4. Comparison of teamwork score between nurses with different educational levels and smoking status $(n=194)$.

\section{Discussion}

We hypothesized that the levels of problem solving and interpersonal communication are regulated by appraisal of other nurses' emotions and utilization of emotions in facing obstacles in clinical settings. The results of this study did not approve our first hypothesis. This is because the study has indicated that the interpersonal communication and problem-solving abilities of the nurses were enhanced with an increasing level of appraisal of their own emotions. However, the study approved our second hypothesis as the level of problem solving was improved with the greater utilization of emotion. The stress level of the nurses also increased with a decreasing level of utilization of emotion.

\subsection{First hypothesis: appraisal of one's own and others' emotions in relation to communication skills}

A cross-sectional study was conducted in 2 populations comprising patients admitted to 3 hospitals. The study showed that nurses' job satisfaction and communication skills have a mediating role in El and service quality. ${ }^{17}$ In other words, nurses with a high capacity of intelligence were expected to deliver more qualified health care and health-related services. In agreement with this study, we showed that interpersonal communication, including expressions of enthusiasm for nursing work, cooperating with the supervisor nurse, and behaving in a friendly manner, was improved with an appraisal of one's own emotions. In addition, the problem-solving abilities of the nurses, including identifying sudden changes related to the patient's condition, quickly solving clinical problems, and taking the initiative to solve a work-related problem, improved with the enhanced appraisal of their own emotions. It seems that when the nurses critique their own emotions and those of others, they establish a basis of understanding to identify those styles that are more suitable in communicating with other nurses or their supervisors. ${ }^{18}$

We did not examine the mediating role of communication skills in the relation between El and service quality since service quality was not measured in this study. However, El is important to nurses owing to its effect on communication skills. Importantly, the perceptions of patients toward their health care highly depend on the quality of their interactions with their 


\begin{tabular}{|c|c|c|c|c|c|c|c|c|}
\hline \multirow{2}{*}{ Control variables } & \multicolumn{2}{|c|}{ Interpersonal communication } & \multicolumn{2}{|c|}{ Problem solving } & \multicolumn{2}{|c|}{ Teamwork } & \multicolumn{2}{|c|}{ Stress score } \\
\hline & $r$ & $P$ & $r$ & $P$ & $r$ & $P$ & $r$ & $P$ \\
\hline Appraisal of own emotions & -0.263 & $<0.001$ & -0.241 & 0.001 & & & & \\
\hline $\begin{array}{l}\text { Regulation of others' } \\
\text { emotions }\end{array}$ & -0.591 & $<0.001$ & & & -0.527 & $<0.001$ & & \\
\hline Utilization of emotions & & & -0.358 & $<0.001$ & & & 0.368 & $<0.001$ \\
\hline
\end{tabular}

Note: The analyses were adjusted for nurse age, nurse gender, marital status, residency, smoking, physical activity, sleeping hours, number of family members, and stress score.

Table 5. Correlation of dimensions of emotional intelligence with clinical performance and stress.

health care providers, including nurses, doctors, and the medical team. ${ }^{19,20}$ Furthermore, the connection that a patient feels with a clinician can ultimately enhance their health status by promoting participation in their care, adherence to therapy, and patient self-management. ${ }^{20-23}$

Derived from Gardner's ${ }^{24}$ idea of multiple intelligences, El involves an individual's ability to "monitor their own and others' emotions, to discriminate among them, and to use the information to guide thinking and actions." Hence, we can understand from the nurses who participated in this study that they appraised their own and others' abilities to appropriately communicate with other nurses, patients, and supervisors. But we hypothesized that appraising others' emotions has a role in regulating interpersonal communication with patients and nursing supervisors. The relationship between El and clinical skills has been reported elsewhere. ${ }^{25}$ Stratton et al. ${ }^{25}$ have indicated that the "attention to feeling" subscale of El and the "perspective-taking" and "empathic concern" subscales of empathy were associated with communication skills among clinical students. In this case, the "attention to feelings" subscale assessed the extent to which the student adhered to the verbal and nonverbal appraisal and expression of emotions. Accordingly, an improvement in clinical skills could result in enhanced teamwork in nurses.

Self-awareness provides a road map for a person to adjust the job, motivate oneself and others, and assess the feelings of others to effectively lead and motivate others. ${ }^{26}$ People with the abilities of self-awareness and appraisal of others' emotions can recognize and identify what their emotions are and how to apply these emotions to improve their relationships with other individuals. ${ }^{27}$

We showed in this study that communication skills can be managed through enhancing the skills of nurses in appraising their own emotions. But this kind of relationship was not found for the appraising of others' emotions. We refer to this as a weakness of the nurses because the nurses who participated in this study were unable to appraise the emotions of other nurses.

\subsection{Second hypothesis: regulation of others' emotions in relation to teamwork}

We found that teamwork in nurses was improved through the regulation of others' emotions in agreement with our hypothesis. The teamwork in this study included cooperating with the other members of the team, engaging responsibly in meetings and group activities, giving feedback to colleagues in a constructive way, and engaging in and contributing to research-based practices. Cadman and Brewer ${ }^{28}$ have determined the key El competencies required for nurses to succeed in health settings. These competencies are the ability to work collaboratively with interdisciplinary teams, the ability to recognize and respond to one's own and others' emotions (self-awareness), and the ability to motivate other nurses in clinical aspects. ${ }^{28}$ Emotional competencies have been defined as critical for the future success of nurses in health settings. Strickland ${ }^{29}$ determined the following emotional competencies as essential for the nursing profession: "self-awareness, self-management and emotional self-control, [and] relationship management competencies such as empathy and teamwork." These competencies lead to increased profitability, patient satisfaction, motivation, and cooperation of co-workers. ${ }^{29}$

\subsection{Emotion appraisal and utilization in relation to problem solving}

The present study found that competence in problem solving improved with improvements in the abilities of nurses to appraise and utilize their own emotions. The problem solving items were assessing their ability to identify sudden changes related to the patient's condition, solving speedy clinical issues, and taking the initiative to solve a work-related problem. $\mathrm{RaO}^{30}$ reported that accurate appraisal and discernment of patient emotions have a positive effect on the quality and accuracy of history taking and diagnosis. When medical practitioners can better understand the emotional reactions of patients and make recommendations for therapeutic plans and lifestyle modifications, they can understand 
why some interventions are more or less suitable for patients and make a suitable intervention plan. The effectiveness of emotion appraisal and its utilization in problem solving, especially for patients with medical conditions, could be due to its effect on burnout. ${ }^{31}$

Akerjordet and Severinsson ${ }^{32}$ reported that $\mathrm{EI}$ in nurses is associated with more positive attitudes, greater adaptability, and improvement in the relationship between nursing staff and their patients. El correlates with problem solving in nurses because higher $\mathrm{El}$ is associated with higher improved overall cognitive-based performance that is essential for problem solving. ${ }^{33}$ Also, higher El competence is associated with decreasing stress levels $^{34}$, as also shown in this study.

El dimensions were not correlated with leadership skills in this study. The leadership skills include motivating other nurses, coaching others in duties, having supervisor attributes, and helping beginning-level nurses with their entry to practice. The possible reason is the nurses who were included in this study had similar levels of $\mathrm{El}^{3}$ since they were hesitant about assessing leadership in terms of clinical performance $(M=3.76$; $S D=0.82)$. Another study has shown that nurse supervisors with high El scores can adapt their leadership style to meet the needs of the staff. ${ }^{35}$ Other similar investigations on nursing leadership and El have reported that $\mathrm{El}$ is positively associated with staff adaptation in terms of change and the improvement of interdisciplinary links and patient satisfaction. ${ }^{36,37}$

\section{Conclusions}

The present investigation showed that the appraisal of one's own emotions improves interpersonal

\section{References}

1. Kamau C, Medisauskaite A, Lopes, B. Inductions buffer nurses' job stress, health, and organizational commitment. Arch Environ Occup Health. 2015;70:305-308.

2. Sharma P, Davey A, Davey S, et al. Occupational stress among staff nurses: controlling the risk to health. Indian J Occup Environ Med. 2014;18:52.

3. Ali YY, Morad AD, Sabri PR. Emotional intelligence dimensions as predictors of coping reactions to stress in nursing practitioners. Fukushima $J$ Med Sci. 2020;65:99-108.

4. Salovey P, Grewal D. The science of emotional intelligence. Curr Dir Psychol Sci. 2005;14:281-285.

5. VandenBos GR. APA Dictionary of Psychology. 2nd ed. Washington, DC: American Psychological Association; 2015:1204. communication and problem-solving skills in nurses. The appraisal of others' emotions improves interpersonal communication and teamwork. Besides, the utilization of emotions enhances nurses' problem-solving abilities concerning medical issues.

\section{Recommendations}

We suggest that nurses be educated on how to regulate others' emotions and positively utilize these emotions to face obstacles in medical settings. But we need to take into consideration the limitation that El cannot be quickly developed exclusively through interpersonal skill training. Therefore, it is required that nurse educators develop assessment strategies to identify $\mathrm{El}$ in the recruitment process. ${ }^{28}$ In other words, nurses need to know how to regulate others' emotions and positively utilize these emotions to make a positive communication in medical settings.

\section{Ethical approval}

The ethical approval of the present study was obtained from the local health ethics committee in Duhok with the following registered reference number: 12072017-5 (on July 12, 2017). The authors obtained a written consent form from all nurses. The nurses' right to refuse participation was protected in this study. A guarantee of confidentiality of the obtained information was given to nurses at the time of publication.

\section{Conflicts of interest}

All authors have nothing to disclose.
6. Tofthagen R, Fagerstrøm LM. Rodgers' evolutionary concept analysis - a valid method for developing knowledge in nursing science. Scand $J$ Caring Sci. 2010;24:21-31.

7. Vahidi $M$, Namdar Areshtanab $H$, Arshadi Bostanabad M. The relationship between emotional intelligence and perception of job performance among nurses in North West of Iran. Scientifica (Cairo). 2016;2016:9547038.

8. McQueen AC. Emotional intelligence in nursing work. J Adv Nurs. 2004;47:101-108.

9. Farshi MR, Vahidi M, Jabraeili M. Relationship between emotional intelligence and clinical competencies of nursing students in Tabriz Nursing and Midwifery School. Res Dev Med Educ. 2014;4:91-95. 
10. Beauvais AM, Brady N, O'Shea ER, et al. Emotional intelligence and nursing performance among nursing students. Nurse Educ Today. 2011;31: 396-401.

11. Chew B-H, Md. Zain A, Hassan F. The relationship between the social management of emotional intelligence and academic performance among medical students. Psychol Health Med. 2015;20:198-204.

12. Moslehi M, Samouei R, Tayebani T, et al. A study of the academic performance of medical students in the comprehensive examination of the basic sciences according to the indices of emotional intelligence and educational status. J Educ Health Promot. 2015;4:66.

13. Davies KA, Lane AM, Devonport TJ, et al. Validity and reliability of a brief emotional intelligence scale (BEIS-10). J Individ Differ. 2010;31:198-208.

14. Harden R. What is an OSCE? Med Teach. 1988;10:19-22.

15. Ross M, Carroll G, Knight J, et al. Using the OSCE to measure clinical skills performance in nursing. J Adv Nurs. 1988;13:45-56.

16. Cohen S. Perceived stress scale. 1994. http://www. mindgarden.com/documents/PerceivedStressScale.pdf. Accessed December 21, 2020.

17. Ezzatabadi MR, Bahrami MA, Hadizadeh F, et al. Nurses' emotional intelligence impact on the quality of hospital services. Iran Red Crescent Med J. 2012;14:758.

18. Shih HA, Susanto E. Conflict management styles, emotional intelligence, and job performance in public organizations. Int $J$ Confl Manage. 2010;21:147-168.

19. Wanzer MB, Booth-Butterfield M, Gruber K. Perceptions of health care providers' communication: relationships between patient-centered communication and satisfaction. Health Commun. 2004;16:363-384.

20. Duffy FD, Gordon GH, Whelan G, et al. Assessing competence in communication and interpersonal skills: the Kalamazoo II report. Acad Med. 2004;79:495-507.

21. Heisler M, Bouknight RR, Hayward RA, et al. The relative importance of physician communication, participatory decision making, and patient understanding in diabetes self-management. J Gen Intern Med. 2002;17:243-252.

22. Safran DG, Taira DA, Rogers WH, et al. Linking primary care performance to outcomes of care. J Fam Pract. 1998;47:213-220.

23. Zachariae R, Pedersen CG, Jensen AB, et al. Association of perceived physician communication style with patient satisfaction, distress, cancer-related self-efficacy, and perceived control over the disease. Br J Cancer. 2003;88:658-665.

24. Gardner H. Frames of Mind: The Theory of Multiple Intelligences. UK: Hachette; 2011.

25. Stratton TD, Elam CL, Murphy-Spencer AE, et al. Emotional intelligence and clinical skills: preliminary results from a comprehensive clinical performance examination. Acad Med. 2005;80: S34-S37.

26. Gordon-Handler L. The Relationship Between Emotional Intelligence and Clinical Performance in an Occupational Therapy Training Program. Minneapolis: Northcentral University; 2009.

27. Salovey PE, Sluyter DJ, Emotional Development and Emotional Intelligence: Educational Implications. New York: Basic Books; 1997.

28. Cadman C, Brewer J. Emotional intelligence: a vital prerequisite for recruitment in nursing. J Nurs Manage. 2001;9:321-324.

29. Strickland D. Emotional intelligence: the most potent factor in the success equation. J Nurs Admin. 2000;30:112-117

30. Rao PR. Emotional intelligence: the sine qua non for a clinical leadership toolbox. J Commun Disord. 2006;39:310-319.

31. Gerits L, Derksen JJ, Verbruggen AB. Emotional intelligence and adaptive success of nurses caring for people with mental retardation and severe behavior problems. Ment Retard. 2004;42: 106-121.

32. Akerjordet K, Severinsson E. Emotional intelligence: a review of the literature with specific focus on empirical and epistemological perspectives. J Clin Nurs. 2007;16:1405-1416.

33. Lam LT, Kirby SL. Is emotional intelligence an advantage? An exploration of the impact of emotional and general intelligence on individual performance. J Soc Psychol. 2002;142:133-143.

34. Pau A, Rowland ML, Naidoo S, et al. Emotional intelligence and perceived stress in dental undergraduates: a multinational survey. J Dent Educ. 2007;71:197-204.

35. Mccallin A, Bamford A. Interdisciplinary teamwork: is the influence of emotional intelligence fully appreciated? J Nurs Manage. 2007;15:386-391.

36. Cummings G, Hayduk L, Estabrooks C. Mitigating the impact of hospital restructuring on nurses: the responsibility of emotionally intelligent leadership. Nurs Res. 2005;54:2-12.

37. Snow JL. Looking beyond nursing for clues to effective leadership. J Nurs Admin. 2001;31:440-443. 